\title{
Evaluation of destruction in a collagen-induced arthritis rat model: Bony spur formation
}

\author{
YIPING HU ${ }^{1,2^{*}}$, YI YANG ${ }^{3 *}$ and BIN LUO ${ }^{4 *}$ \\ ${ }^{1}$ Center for Translational Medicine Research and Development, Shenzhen Institute of Advanced Technology, \\ Chinese Academy of Science; ${ }^{2}$ Shenzhen College of Advanced Technology, University of Chinese Academy \\ of Sciences, Shenzhen, Guangdong 518055; Departments of ${ }^{3}$ Gynecology and ${ }^{4}$ Cardiac and Vascular \\ Surgery, Shenzhen People's Hospital, Jinan University, Shenzhen, Guangdong 518020, P.R. China
}

Received April 29, 2016; Accepted April 21, 2017

DOI: $10.3892 /$ etm.2017.4817

\begin{abstract}
Over the past 40 years, the collagen-induced arthritis (CIA) animal model has been widely used as a model of rheumatoid arthritis (RA). However, no model is able to completely depict the characteristics of cartilage destruction to date. In the later stage of joint cartilage destruction, bony spurs form in RA. This bony spur formation is an important symptom in the pathological development of RA. In the present study, CIA was used to elucidate the pathological process of bony spur formation. Joint damage and spur formation in the animal model was detected by radiology and histology. Radiology identified bony spurs in the knee and foot joints, which worsened as the disease progressed. Furthermore, following observations of histological sections, fusion and damage of the articular cartilage, as well as a higher number of osteoclasts, were identified. Previous results have determined that bony spurs may be involved in another pathological process that occurs during the later stages of RA. Therefore, further studies investigating this symptom are required to improve the understanding of RA and facilitate the development of an appropriate treatment for RA.
\end{abstract}

Correspondence to: Dr Yiping Hu, Center for Translational Medicine Research and Development, Shenzhen Institute of Advanced Technology, Chinese Academy of Science, 1068 Xueyuan Avenue, Shenzhen, Guangdong 518055, P.R. China

E-mail: hover126@126.com

Dr Bin Luo, Department of Cardiac and Vascular Surgery, Shenzhen People's Hospital, Jinan University, 1017 East Gate North Road, Shenzhen, Guangdong 518020, P.R. China

E-mail: luobin720515@126.com

*Contributed equally

Key words: rheumatoid arthritis, collagen-induced arthritis, bony spur

\section{Introduction}

Rheumatoid arthritis (RA) is a chronic autoimmune disease. The possible causes of RA remain unclear at present, which renders it difficult to treat. A number of strategies have been used to treat RA that target different pathological stages of the disease $(1,2)$. The primary symptom of RA is inflammation of the synovial lining of the articular cartilage. Therefore, the majority of therapeutic strategies for RA inhibit inflammatory cytokines, such as tumor necrosis factor (TNF) (3). However, none of the current treatments completely cure RA, therefore further studies are required to determine its exact pathology and enable the development of curative treatments.

Long-term and systemic chronic inflammation leads to the development of lesions in the joint architecture, which is the structural basis for progressive impairment of function. The primary radiographic characteristics of RA include articular cartilage inflammation, peri-articular bone loss, uniform joint space loss, bone destruction and soft-tissue swelling of the entire joint (4). Sustained inflammatory joint erosion may be accompanied by the formation of bony spurs, also known as osteophytes. These bony spurs form at the joint edges in various diseases, including psoriatic arthritis and ankylosing spondylitis (5). The causes of the divergent bone responses in various inflammatory diseases are currently unknown but may involve the differential regulation of local bone homeostasis in the course of inflammatory arthritis (6).

The aforementioned previous studies suggest that bony spur formation is primarily a result of articular damage. Osteoclasts are considered to be the primary bone-resorbing cell in inflammatory arthritis and may serve a key role in RA $(7,8)$. Bony spur formation is an important symptom of inflammatory joint disease, due to inflammation leading to joint degeneration, such as articular cartilage thinning and joint space narrowing. Abnormal cartilage and bone proliferation cause spur formation and chronic pain (9), however its mechanism of action is currently not well understood. Thus, increased attention should be given to bony spur formation associated with inflammatory cytokines, bone metabolic syndrome and molecules (such as glucocorticoids). Improving these symptoms may affect the formation of bony spurs and modify structural joint damage $(10,11)$. 
Therefore, the current study aimed to evaluate bony spur formation in a collagen-induced arthritis (CIA) animal model and investigate the role of bony spurs in the process of articular cartilage damage. The results of the current study may therefore aid future research into the pathology of RA.

\section{Materials and methods}

Animals. Wistar rats (25 female rats, 8-10 weeks old, mean weight $180 \mathrm{~g}$ ) were purchased from Vital River Laboratory Animal Technology Co., Ltd (Beijing, China). Rats were kept and fed in specific pathogen-free conditions at the animal facility of Shenzhen Institute of Advanced Technology, Chinese Academy of Sciences (Shenzhen, China). All animals were housed in cages with a 12-h light/dark cycle, $60 \%$ humidity and room temperature of $21^{\circ} \mathrm{C}$. All procedures performed in the present study were approved by the Institutional Animal Care and Use Committee of the Shenzhen Institute of Advanced Technology.

Induction of CIA in rats. Randomly selected rats $(\mathrm{n}=20)$ were immunized with collagen type II (CII), and 5 normal rats were not immunized as the control. CIA was induced using the method described by Trentham et al (12). In brief, bovine collagen-II (CII) (CII; Chondrex Inc., Redmond, WA, USA) was dissolved in $0.05 \mathrm{M}$ acetic acid and emulsified with isovolumic incomplete Freund's adjuvant (IFA; 7,002, Chondrex Inc.). A total of $0.2 \mathrm{ml}$ CII-IFA emulsification solution was administered as an intradermal injection at the tail of each rat; the injection site was $\sim 2 \mathrm{~cm}$ away from the tail root. An additional $0.1 \mathrm{ml} \mathrm{CII-IFA} \mathrm{emulsification} \mathrm{solution} \mathrm{was}$ subcutaneously injected as a booster immunization into the opposite side of the tail 10 days after the initial immunization. All rats were observed 3 times a week by the same investigator to assess arthritis. The incidence of arthritis and the clinical score were evaluated.

When arthritis symptoms were present a semi-quantitative scoring system was used to assess the severity of arthritis as follows: 0 , normal joint; 1 , swelling and redness in 1 joint; 2 , swelling in $>1$ joint; 3 , whole-paw swelling and 4 , joint deformity and/or ankylosis (13). Accumulated scores for all four paws of each rat (maximum possible score of 16) were used to determine disease severity and progression. To evaluate disease incidence, animals were considered to have arthritis if the score increased $\geq 2$ points compared with the score at the start of the experiment.

Radiological analysis. X-rays at $35 \mathrm{kV}$ and $20 \mathrm{sec}$ (Faxitron Bioptics, LLC, Tucson, AZ, USA) and micro-computed tomography (micro-CT; 1,076 Bruker microct, Kontich, Belgium) scans of the lateral view were recorded at $60 \mathrm{kV}$ and $400 \mu \mathrm{A}$, at 3 months following the second immunization.

Histological analysis. Rats were sacrificed using an overdose $(100 \mathrm{mg} / \mathrm{kg}$ ) of pentobarbital sodium salt (Merck KGaA, Darmstadt, Germany) and claws were fixed in $4.0 \%$ formaldehyde for $12 \mathrm{~h}$ at room temperature prior to decalcification in $10 \%$ EDTA solution at room temperature for $\sim 30$ days. Paraffin sections of the claws $(7 \mu \mathrm{m})$ were stained using a hematoxylin and eosin (H\&E) kit (Nanjing Jiancheng Technology Co., Ltd.,
Nanjing, China) to identify synovial inflammation and bone destruction. A leukocyte acid phosphatase staining (TRAP) kit (Sigma-Aldrich, Inc., Merck KGaA) was used to detect osteoclasts, according to the manufacturer's instructions. Sections were observed using an Olympus BX53 microscope (Olympus Corporation, Tokyo, Japan).

Statistical analysis. To evaluate the effects of different treatments changes in the arthritis scores between the beginning and end of the experiment was determined in rats from each group. Statistical significance was determined using a Kruskal-Wallis test. SPSS 17.0 was used to assess the results of all experiments (SPSS, Inc., Chicago, IL, USA) and the statistical significance level was set at $\mathrm{P}<0.05$.

\section{Results}

CIA model. Two rats presented with symptoms of arthritis 5 days after the second immunization whereas the majority of the rats exhibited this symptom $\sim 10$ days after the second immunization (CIA symptoms were observed in 12 of 20 immunized rats). Macroscopic pathology indicated redness and swelling in the joints of the CIA model rats (Fig. 1). The redness and swelling of the joints of CIA model rats (Fig. 1B) was notably higher than that of control rats (Fig. 1A). The results of the semi-quantitative scoring system demonstrated that this difference in arthritis severity was significant $(\mathrm{P}<0.05$; Fig. 1C).

Emergence of bony spur formation following synovial inflammation and bone destruction. Radiology results indicated that bony spur formation accompanied with joint destruction was observed 3 months after the second immunization in the CIA model rats (Fig. 2A). By contrast, no bony spur formation was detected in the control rats (Fig. 2B). A 3D micro-CT analysis demonstrated that the joint damage in the CIA model group was severe and that the original form of the joint was almost invisible (Fig. 2C). This was not evident in control rats (Fig. 2D). H\&E staining of the joint revealed that bony spur formation was accompanied by articular cartilage damage (Fig. 3A). Furthermore, TRAP staining demonstrated that TRAP-positive cells adhered to the eroded surface of the bony area (Fig. 3B). These osteoclast cells directly contributed to the erosion of cartilage and these results indicate that the differentiation status of chondrocytes in the CIA model was altered in an inflammatory environment, similar to the histological expression of endochondral ossification.

\section{Discussion}

Information regarding bony spur formation, considered to be an important symptom of human RA, is currently limited. Thus, further studies are required to investigate bony spur formation. Bony spur formation is a common pathological symptom in inflammatory and degenerative joint diseases and these bony spurs usually form at the joint edge or at the insertion sites of tendons (6). Previous results have suggested that the formation of bony spurs may represent a response-to-stress mechanism in joint disease (14). Furthermore, mechanical stress (such as load and friction), 

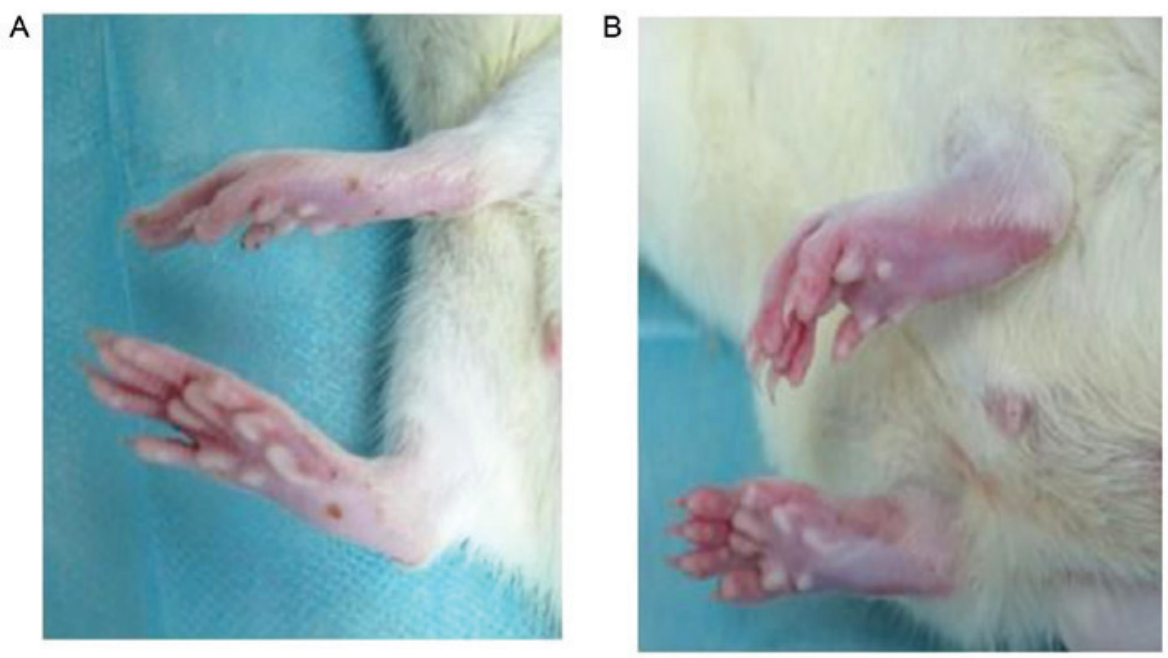

C

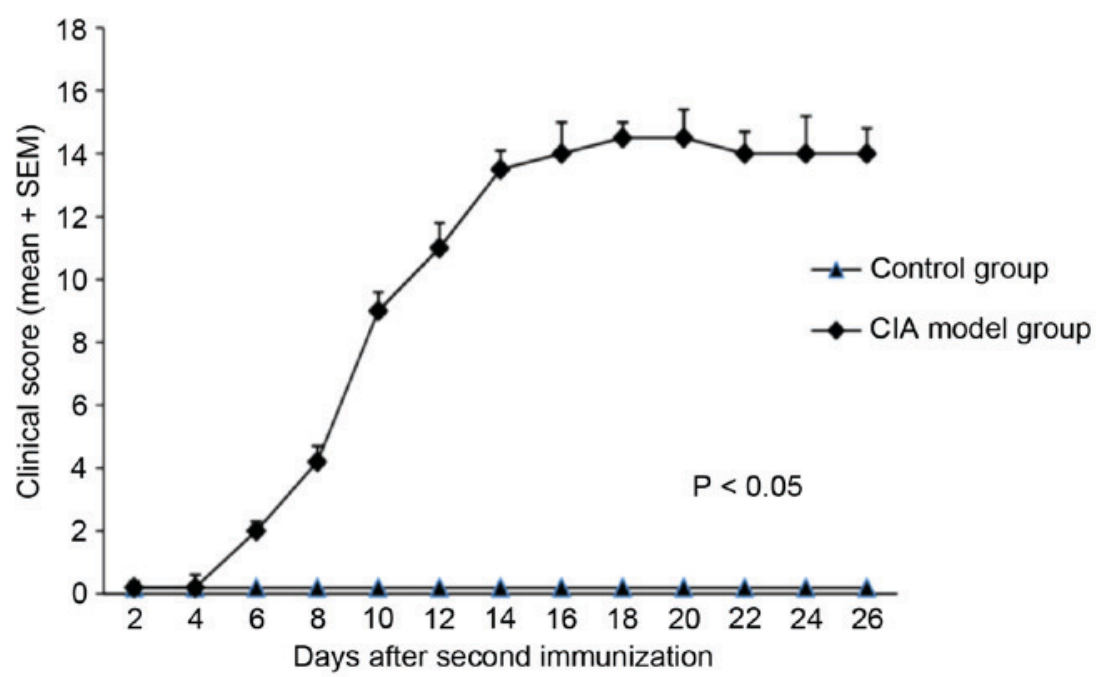

Figure 1. Macroscopic pathology of joint swelling in rats with CIA. (A) Control group (n=5), (B) arthritis onset in the CIA group (10 of the 15 induced rats revealed symptoms of rheumatoid arthritis) and (C) the clinical scoring indicating arthritis severity. Differences between the control and the model animal group were statistically significant $(\mathrm{P}<0.05)$. CIA, collagen-induced arthritis; SEM, standard error of the mean.

metabolic discord, subchondral bone sclerosis and long-term inflammation may lead to the formation of bony spurs. In RA, mechanical and inflammatory triggers are particularly important factors influencing bony spur formation. Arthritis patients experience persistent stress and long-term inflammatory symptoms, these pathologies support the conclusion that bony spur formation is a chronic process that is accompanied by joint pain (15). Furthermore, bony spurs may be a response to joint stress and this pathology may also contribute to the disease burden, as it leads to stiffness of the entire joint and the loss of motion (16).

These insights support the hypothesis that bony spur formation depends on molecules associated with bone formation, including transforming growth factor $\beta$, bone morphogenetic proteins and the Wnt protein family $(17,18)$. To improve understanding of bony spur formation, studies have established animal models of human inflammatory joint disease, which have the potential to form bony spurs. Different models of inflammatory arthritis, such as CIA, determine therapeutic intervention in these inflammatory pathways. Treatment strategies currently applied include suppression of interleukin-1, TNF- $\alpha$ and the immune complex $(19,20)$. Inhibition of these inflammatory cytokines is important to facilitate the improvement of chronic arthritis, however their effect on bony spur formation remains unknown (21). Following the development of an animal model of RA, transgenic and gene-knockout models have been constructed; however, these models do not exactly mimic the disease progression of human RA (22). Although the role of these pathways in the formation of bony spurs and their mutual interaction cannot be confirmed, when joints become inflamed or are subjected to mechanical stress it may activate these essential bone-forming molecular pathways, such as the Wnt/ $\beta$-catenin protein pathway (23).

Osteoclasts are often detected in bony spur areas and cause joint pannus formation that is an important pathological feature of arthritis (24). The growth of bony spurs requires the formation of endochondral bones, which requires osteoclasts and is characterized by the production of a cartilaginous scaffold containing hypertrophic chondrocytes followed by remodeling into bone $(18,25)$. Furthermore, bony spur formation is associated with an increase in bone metabolism, 

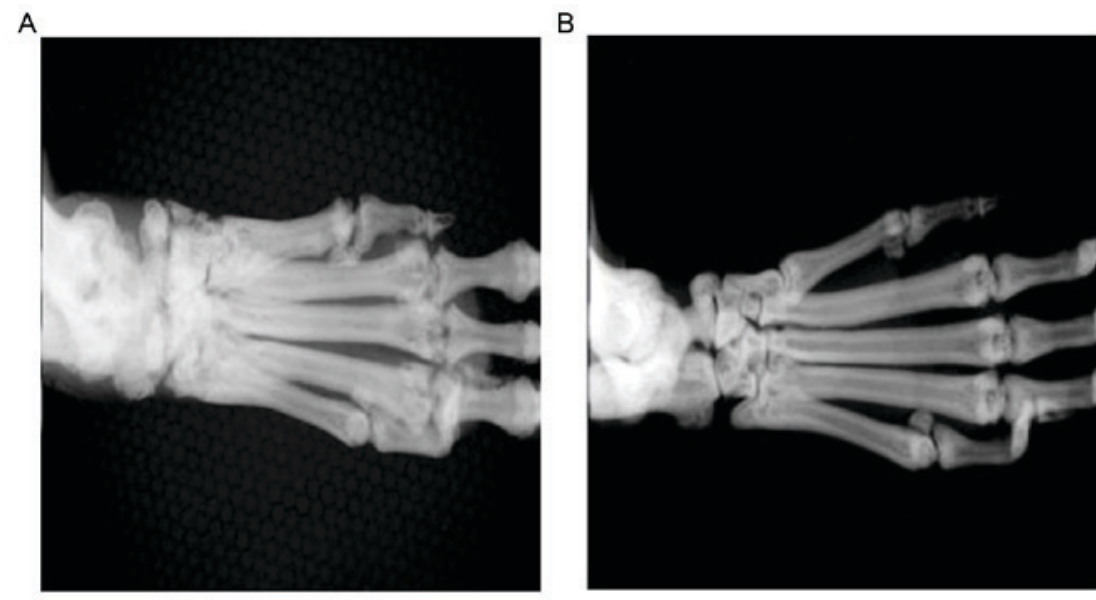

C

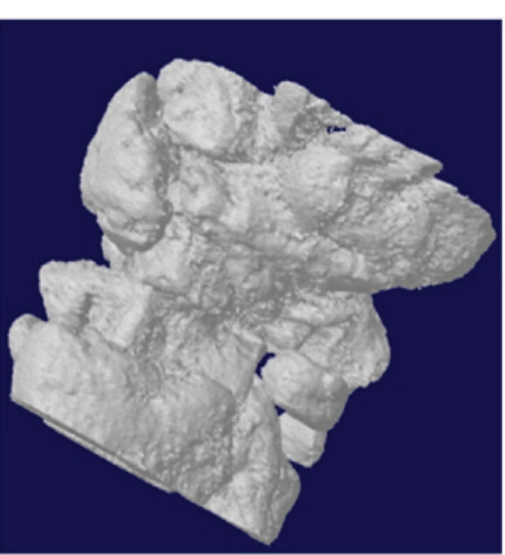

D

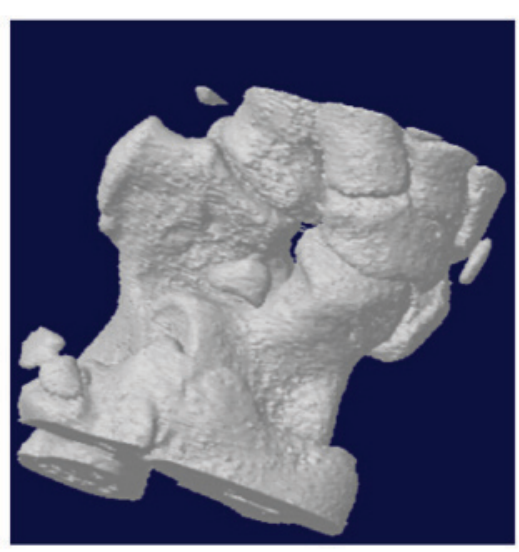

Figure 2. Radiological observation of rats. Evaluation of arthritis onset in the (A) CIA model group and (B) control group. 3D micro-computed tomography image of joint destruction in the (C) CIA model group and (D) control group.
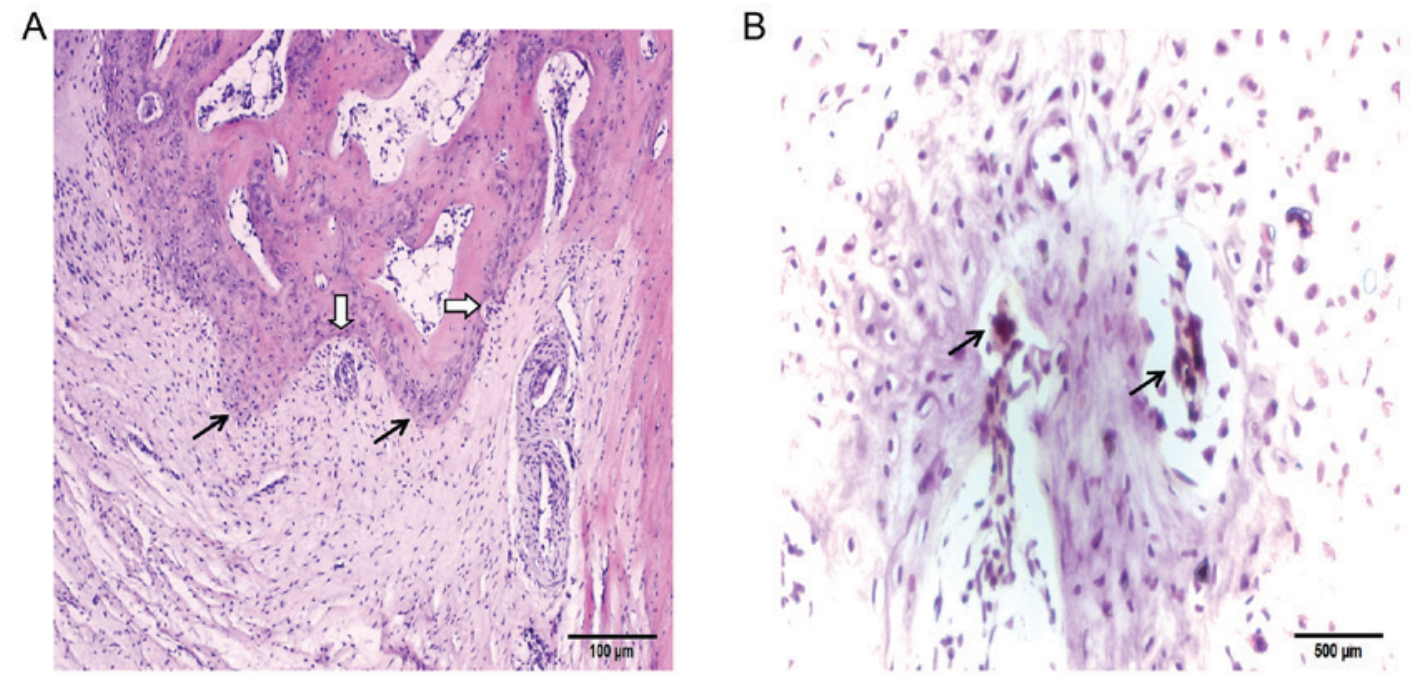

Figure 3. Histological observation of joint bony spur formation and bone damage. (A) Tissue sections were stained with H\&E to study bony spur formation (black arrows) and bone destruction (white arrows). (H\&E stain; original magnification, x100); (B) Osteoclast observation (black arrows), where tissue sections were stained with TRAP (TRAP stain, original magnification, x200). H\&E, hematoxylin and eosin; TRAP, tartrate-resistant acid phosphatase.

with enhanced bone resorption and formation facilitating the construction of newly formed bone (18). Additionally, lesion site osteoclast formation and osteoclast resorption of bone are construed as the primary mechanisms responsible for the catabolic pattern of inflammatory joint damage typically observed in arthritis (26). Therefore, interventions in bone metabolism may affect RA treatment strategies.

In conclusion, the results of the present study demonstrate that articular cartilage fusion is a pathological phenomenon that occurs during the later stage of CIA model development. 
Articular cartilage fusion is similar to endonchondral ossification and may serve an important role in the formation of bony spur. These data indicate that bony spur formation is a response to injury, inflammatory stimuli and bone metabolism, but at present these pathological phenomena are not fully understood. Further in-depth studies are required to determine the underlying mechanisms of bony spur formation.

\section{Acknowledgements}

This work wassupportedbytheScienceandTechnology Innovation Fund of Shenzhen (grant nos. JCYJ20150403101028196, JCYJ20140416122811926 and CXZZ20150529144128031), the Guangdong Science And Technology Planning Project (grant no. 2013B050800005) and the Shenzhen Peacock Plan Foundation (grant no. KQCX2015033117354152).

\section{References}

1. Beirith SC, Ikino CM and Pereira IA: Laryngeal involvement in rheumatoid arthritis. Braz J Otorhinolaryngol 79: 233-238, 2013. (In English, Portuguese).

2. Audo R, Combe B, Hahne M and Morel J: The two directions of TNF-related apoptosis-inducing ligand in rheumatoid arthritis. Cytokine 63: 81-90, 2013.

3. Zhang P, Han D, Tang T, Zhang X and Dai K: Inhibition of the development of collagen-induced arthritis in Wistar rats through vagus nerve suspension: A 3-month observation. Inflamm Res 57: 322-328, 2008.

4. Jacobson JA, Girish G, Jiang Y and Resnick D: Radiographic evaluation of arthritis: Inflammatory conditions. Radiology 248: 378-389, 2008.

5. Schett G, Landewé R and van der Heijde D: Tumour necrosis factor blockers and structural remodelling in ankylosing spondylitis: What is reality and what is fiction? Ann Rheum Dis 66: 709-711, 2007.

6. Schett G, Stolina M, Dwyer D, Zack D, Uderhardt S, Krönke G, Kostenuik $\mathrm{P}$ and Feige U: Tumor necrosis factor alpha and RANKL blockade cannot halt bony spur formation in experimental inflammatory arthritis. Arthritis Rheum 60: 2644-2654, 2009.

7. Redlich K, Hayer S, Ricci R, David JP, Tohidast-Akrad M, Kollias G, Steiner G, Smolen JS, Wagner EF and Schett G: Osteoclasts are essential for TNF-alpha-mediated joint destruction. J Clin Invest 110: 1419-1427, 2002.

8. Lam J, Takeshita S, Barker JE, Kanagawa O, Ross FP and Teitelbaum SL: TNF-alpha induces osteoclastogenesis by direct stimulation of macrophages exposed to permissive levels of RANK ligand. J Clin Invest 106: 1481-1488, 2000.

9. Al-Jarallah K, Shehab D, Abdella N, Al Mohamedy H and Abraham M: Knee osteoarthritis in type 2 diabetes mellitus: Does insulin therapy retard osteophyte formation? Med Princ Pract 25: 12-17, 2016.

10. Diarra D, Stolina M, Polzer K, Zwerina J, Ominsky MS, Dwyer D, Korb A, Smolen J, Hoffmann M, Scheinecker C, et al: Dickkopf-1 is a master regulator of joint remodeling. Nat Med 13: 156-163, 2007.
11. Rostom S, Mengat M, Lahlou R, Hari A, Bahiri R and Hajjaj-Hassouni N: Metabolic syndrome in rheumatoid arthritis: Case control study. BMC Musculoskelet Disord 14: 147, 2013.

12. Trentham DE, Townes AS and Kang AH: Autoimmunity to type II collagen an experimental model of arthritis. J Exp Med 146: 857-868, 1977.

13. van Holten J, Reedquist K, Sattonet-Roche P, Smeets TJ, Plater-Zyberk C, Vervoordeldonk MJ and Tak PP: Treatment with recombinant interferon-beta reduces inflammation and slows cartilage destruction in the collagen-induced arthritis model of rheumatoid arthritis. Arthritis Res Ther 6: R239-R249, 2004.

14. Li J and Muehleman C: Anatomic relationship of heel spur to surrounding soft tissues: Greater variability than previously reported. Clin Anat 20: 950-955, 2007.

15. Yalcin E, Keskin Akca A, Selcuk B, Kurtaran A and Akyuz M: Effects of extracorporal shock wave therapy on symptomatic heel spurs: A correlation between clinical outcome and radiologic changes. Rheumatol Int 32: 343-347, 2012.

16. Sieper J, Appel H, Braun J and Rudwaleit M: Critical appraisal of assessment of structural damage in ankylosing spondylitis: Implications for treatment outcomes. Arthritis Rheum 58: 649-656, 2008.

17. Lories RJ, Derese I and Luyten FP: Modulation of bone morphogenetic protein signaling inhibits the onset and progression of ankylosing enthesitis. J Clin Invest 115: 1571-1579, 2005.

18. Scharstuhl A, Vitters EL, van der Kraan PM and van den Berg WB: Reduction of osteophyte formation and synovial thickening by adenoviral overexpression of transforming growth factor beta/bone morphogenetic protein inhibitors during experimental osteoarthritis. Arthritis Rheum 48: 3442-3451, 2003.

19. Goldblatt F and Isenberg DA: New therapies for rheumatoid arthritis. Clin Exp Immunol 140: 195-204, 2005.

20. Rogers JL, Serafin DS, Timoshchenko RG and Tarrant TK: Cellular targeting in autoimmunity. Curr Allergy Asthma Rep 12: 495-510, 2012.

21. Juarez M, Filer A and Buckley CD: Fibroblasts as therapeutic targets in rheumatoid arthritis and cancer. Swiss Med Wkly 142: w13529, 2012.

22. Hu Y, Cheng W, Cai W, Yue Y, Li J and Zhang P: Advances in research on animal models of rheumatoid arthritis. Clin Rheumatol 32: 161-165, 2013.

23. O'Shea PJ, Kim DW, Logan JG, Davis S, Walker RL, Meltzer PS, Cheng SY and Williams GR: Advanced bone formation in mice with a dominant-negative mutation in the thyroid hormone receptor $\beta$ gene due to activation of $\mathrm{Wnt} / \beta$-catenin protein signaling. J Biol Chem 287: 17812-17822, 2012.

24. Zoricic S, Maric I, Bobinac D and Vukicevic S: Expression of bone morphogenetic protein and cartilage-derived morphogenetic proteins during osteophyte formation in humans. J Anat 202: 269-277, 2003.

25. Merry K, Dodds R, Littlewood A and Gowen M: Expression of osteopontin mRNA by osteoclasts and osteoblasts in modelling adult human bone. J Cell Sci 104: 1013-1020, 1993.

26. Zhen G, Wen C, Jia X, Li Y, Crane JL, Mears SC, Askin FB, Frassica FJ, Chang W, Yao J, et al: Inhibition of TGF- $\beta$ signaling in mesenchymal stem cells of subchondral bone attenuates osteoarthritis. Nat Med 19: 704-712, 2013. 